\title{
Theoretical and experimental investigation on friction in lubricated line contacts with different materials and textures in presence of wear
}

\author{
DI PUCCIO Francesca ${ }^{a^{*}}$ and CIULLI Enrico ${ }^{b}$
}

Dipartimento di Ingegneria Civile e Industriale, Università di Pisa

\author{
Largo Lazzarino - 56122 Pisa - Italy \\ adipuccio@ing.unipi.it, bciulli@ing.unipi.it
}

Keywords: friction coefficient; wear; line contacts; slide-to-roll ratio.

\begin{abstract}
An experimental investigation on the friction coefficient in line contacts under mixed and boundary lubrication regimes is described. Rectangular contacts between cylindrical specimens and the flat surface of discs of different material and surface roughness combinations were analyzed. Very low Stribeck numbers have been considered, resulting also in low dimensionless film thickness, so that the morphology of the surfaces and the material had a remarking role. In this work, the theoretical procedure for assessing the friction coefficient in the tested cases is described and compared to experimental results. Additionally, wear effects obtained in boundary lubrication conditions are shown. The surface conditions are put in relation with some particular trends of the friction coefficient obtained for certain combinations of materials and roughness.
\end{abstract}

\section{Introduction}

The nature of friction has been widely investigated since Leonardo's studies, recently commented in [1,2]. However, its prediction still remains a difficult issue. One reason can be found in the intrinsic complexity of the phenomenon and in the number of factors affecting the local interactions between two surfaces in contact, as lubrication conditions, material hardness, surface roughness, and so on. Actually, the main limitations appear the lack of a reliable model for describing the lubricant behavior and the surface microgeometry, despite the progresses in the last decade, e.g. $[3,4]$. It should be noted that most of the studies in the literature deals with mixed or ElastoHydrodynamic Lubrication (EHL).

This study describes results of a wide experimental campaign, where specimens of different material, surface roughness and texture were tested, in many different kinematic conditions. The theoretical aspects of the contact are previously introduced in order to have complete indications on the mechanical aspect of the phenomenon. Simple formulas for predicting friction are also applied and compared to experimental results.

\section{Experimental Apparatus and Specimen Description}

Experimental Test Rig. The apparatus employed in this study, shown in Fig.1, was specifically designed for friction force and interferometric film thickness measurements, as described in $[5,6]$. Its main feature is the independent and continuous actuation of the two elements in contact by means of two DC electric motors, within the range 0 to $3000 \mathrm{rpm}$. In this way, different slide-to-roll ratios (SRR) can be achieved. Additionally, pure rolling conditions can be obtained by simply disconnecting the disc from its actuator. The apparatus enables also the application of different loads by varying the weights shown on the left in Fig.1.

It can be worth noting that the disc and the specimen subgroups are supported by gas bearings of integral and radial type, respectively. Moreover, the specimen is connected to a load cell for friction force measurements. The whole structure supporting specimen and motor is placed on a cross table for micrometrical positioning of the specimen. 
A computer aided testing system was developed in Labview both for controlling the electric motors and performing data acquisition and storage. Saved test data include the inlet lubricant temperature, the elements rotational speeds and the traction force acting on the specimen.

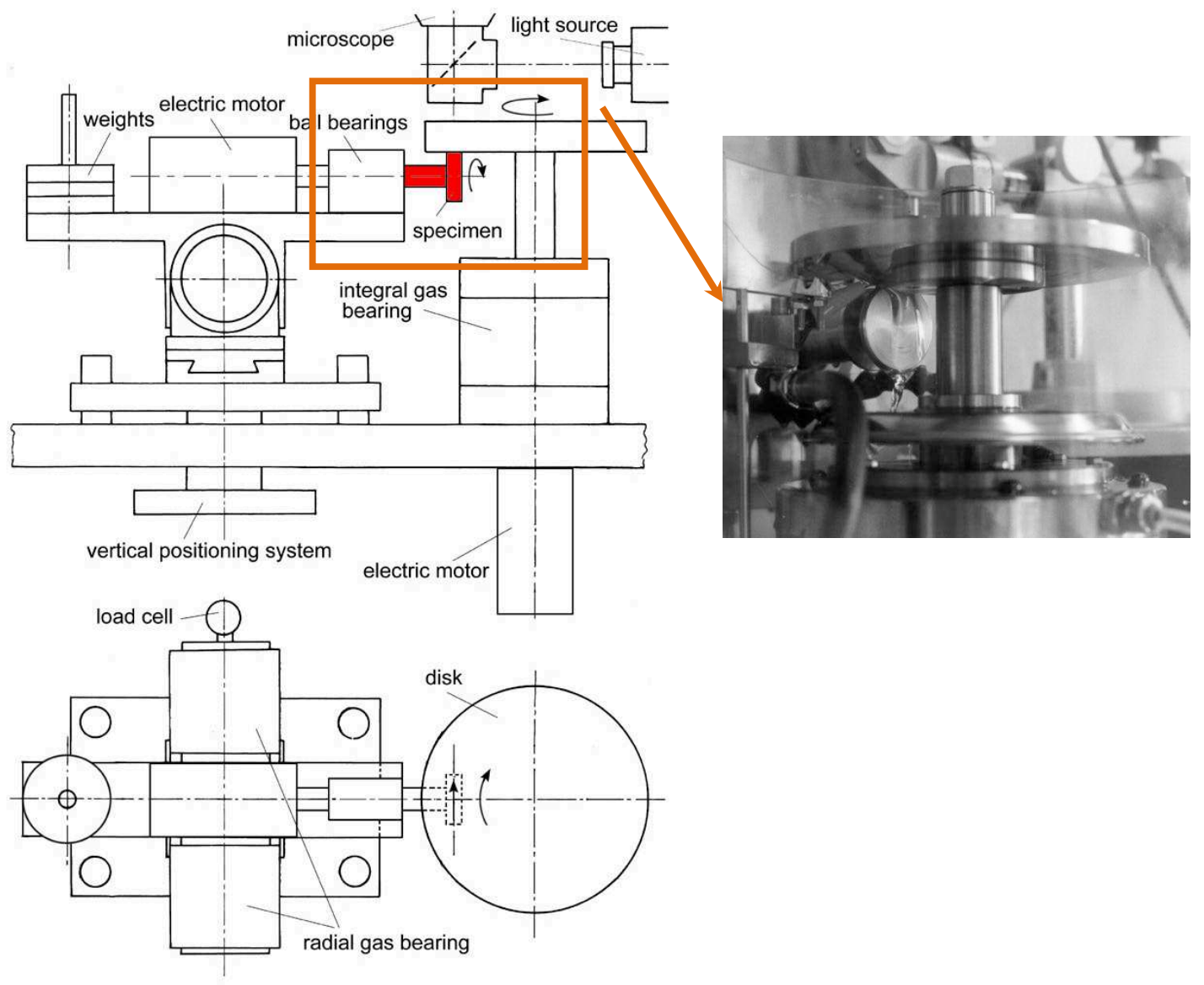

Figure 1: Schematic drawing of the apparatus (left) and picture of the contact detail (right).

Sample Description The specimens adopted in the present test campaign had a cylindrical geometry with a $42 \mathrm{~mm}$ diameter $(R=21 \mathrm{~mm})$ and $3 \mathrm{~mm}$ axial length, while for the disc a $160 \mathrm{~mm}$ diameter was chosen.

In order to investigate the influence of the material properties and he surface roughness on the tribological behaviour of these contact couplings, six discs and six specimens were manufactured. Two discs were made of the aluminum alloy AA6082, (labelled Al), and three of stainless steel X5CrNiMo1810 (labelled St), machined in different ways. The same metals were also used for cylindrical specimens: three made of stainless steel and one of aluminum alloy. Moreover, other two harder materials were examined: a sintered alloy of tungsten carbide and cobalt K40, (Tc) and a kind of steel more suitable for grinding, i.e. $88 \mathrm{MnV} 8,(\mathrm{Sm})$.

Material labels and elastic modulus are collected in Table 1 together with samples ID and their characteristics. In the following, discs and specimens will be identified by labels referring both to their material and surface roughness, e.g. C6-St ${ }_{1800}$ stands for cylinder number 6 made of stainless steel specimen with root mean square roughness $R_{\mathrm{q}} 1.8 \mu \mathrm{m}, \mathrm{D} 1-\mathrm{Al}_{1000}$ is the disk numbered 1 , made of Aluminum alloy, with $R_{\mathrm{q}} 1 \mu \mathrm{m}$ ).

Each cylinder was put in contact with every disc, for a total of 7 material couplings. The effects of the surface roughness were investigated on steel samples (St and $\mathrm{Sm}$ ) assuming 4 different values of the surface roughness for the cylinders and three for the discs and on Aluminim discs with two surface conditions. 
Table 1. Materials and samples properties and labels. $E$ represents the Young modulus and $v$ the Poisson's ratio.

\begin{tabular}{|c|c|c|c|c|c|}
\hline $\begin{array}{c}\text { Material } \\
\text { label }\end{array}$ & $\mathrm{Al}$ & \multicolumn{2}{|c|}{ St } & $\mathrm{Sm}$ & $\mathrm{Tc}$ \\
\hline Material & AA6082 & \multicolumn{2}{|c|}{ X5CRNiMo1810 } & 88MNV8 & $\mathrm{K} 40$ \\
\hline$E$ [GPa] & 70 & \multicolumn{2}{|c|}{210} & 210 & 580 \\
\hline v & 0.33 & \multicolumn{2}{|c|}{0.27} & 0.27 & 0.22 \\
\hline Short ID & D1 & D2 & D3 & D4 & D5 \\
\hline ID & $\mathrm{D} 1-\mathrm{Al}_{1000}$ & $\mathrm{D} 2-\mathrm{Al}_{250}$ & D3-St 1800 & $\mathrm{D} 4-\mathrm{St}_{700}$ & D5-St 130 \\
\hline $\begin{array}{c}\text { Material } \\
\text { label }\end{array}$ & $\mathrm{Al}$ & $\mathrm{Al}$ & St & St & St \\
\hline $\boldsymbol{R}_{q}[\mu \mathrm{m}]$ & 1 & 0.25 & 1.8 & 0.7 & 0.13 \\
\hline Machining & facing & facing & facing & grinding & grinding \\
\hline $\begin{array}{c}\text { Hardness } \\
{[\mathrm{HV}]}\end{array}$ & 110 & 110 & 135 & 135 & 135 \\
\hline
\end{tabular}

\begin{tabular}{|c|c|c|c|c|c|c|}
\hline \multicolumn{7}{|c|}{ CYLINDERS } \\
\hline Short ID & $\mathrm{C} 1$ & $\mathrm{C} 2$ & $\mathrm{C} 3$ & $\mathrm{C} 4$ & $\mathrm{C} 5$ & C6 \\
\hline ID & $\mathrm{C} 1-\mathrm{Al}_{600}$ & $\mathrm{C} 2-\mathrm{Sm}_{60}$ & $\mathrm{C} 3-\mathrm{Tc}_{35}$ & $\mathrm{C} 4-\mathrm{St}_{1800}$ & $\mathrm{C} 5-\mathrm{St}_{1100}$ & $\mathrm{C} 6-\mathrm{St}_{300}$ \\
\hline $\begin{array}{c}\text { Material } \\
\text { label }\end{array}$ & $\mathrm{Al}$ & $\mathrm{Sm}$ & $\mathrm{Tc}$ & St & St & St \\
\hline $\boldsymbol{R}_{q}[\mu \mathbf{m}]$ & 0.6 & 0.06 & 0.035 & 1.8 & 1.1 & 0.3 \\
\hline Machining & $\begin{array}{l}\text { straight } \\
\text { turning }\end{array}$ & $\begin{array}{c}\text { fine } \\
\text { grinding }\end{array}$ & $\begin{array}{c}\text { fine } \\
\text { grinding }\end{array}$ & $\begin{array}{l}\text { straight } \\
\text { turning }\end{array}$ & grinding & Grinding \\
\hline $\begin{array}{c}\text { Hardness } \\
{[\mathrm{HV}]}\end{array}$ & 110 & 800 & 1300 & 155 & 155 & 155 \\
\hline
\end{tabular}

Testing Conditions. The couples of elements to be tested were put in contact under a $20 \mathrm{~N}$ normal load force, giving a linear distribution $w=20 / 3 \mathrm{~N} / \mathrm{mm}$.

Each contact was tested in different rolling to sliding conditions, and with different speeds. Indicating with $u_{d}$ and $u_{c}$ the speed of points on the contact line of the disc and the cylinder respectively, the tests conditions can be identified by two parameters:

a) The rolling speed $u=\frac{u_{d}+u_{c}}{2}$;

b) The slide-to-roll ratio $S R R=\frac{u_{c}-u_{d}}{u}$.

In particular, the following values were assumed for $u$ and $S R R$ :

$u=0.0125,0.025,0.05,0.075,0.1,0.2 \mathrm{~m} / \mathrm{s}$

$S R R=0.25,0.5,1$

for a total of 18 combinations.

Finally, as lubricant a pure diester was used, the bis(2-ethyl-hexyl)phthalate that, for the given test conditions (test temperature $=33^{\circ} \mathrm{C}$ ), has a viscosity of $\mu_{0}=0.036 \mathrm{~Pa} \mathrm{~s}$ and a pressure-viscosity coefficient $\alpha=1.74 \cdot 10^{-8} \mathrm{~Pa}^{-1}$. 


\section{Theoretical Features of the Contacts}

In order to improve the comprehension of experimental results, some theoretical features of the contact for the tested couplings were calculated.

Stiffness. As far as the material combination is concerned, couplings were classified on the basis the equivalent modulus of the coupling $E$, i.e., according to Hertz's theory,

$$
E^{\prime}=2\left(\frac{1-v_{c}^{2}}{E_{c}}+\frac{1-v_{d}^{2}}{E_{d}}\right)^{-1}
$$

where $E$ is the elastic modulus, $v$ the Poisson's ratio (reported in Table 1) and the subscripts $c$ and $d$ refer to the cylinder and disc properties, respectively. The minimum equivalent modulus was obtained for the Al-Al coupling, so it was taken as a reference for scaling all the other material combinations, as shown in Fig.2. Combinations Al-Steel (Steel includes both St and Sm specimens), Al-Tc, Steel-Steel and Steel-Tc are about 1.5, 2, 3 and 4 times stiffer than Al-Al respectively. Consequently to the different elastic behavior, the pressure profiles of the couplings, according to the Hertzian solution, differ as reported in Fig.2. The maximum pressure in the SteelTc coupling is more than twice higher than in the Al-Al case.

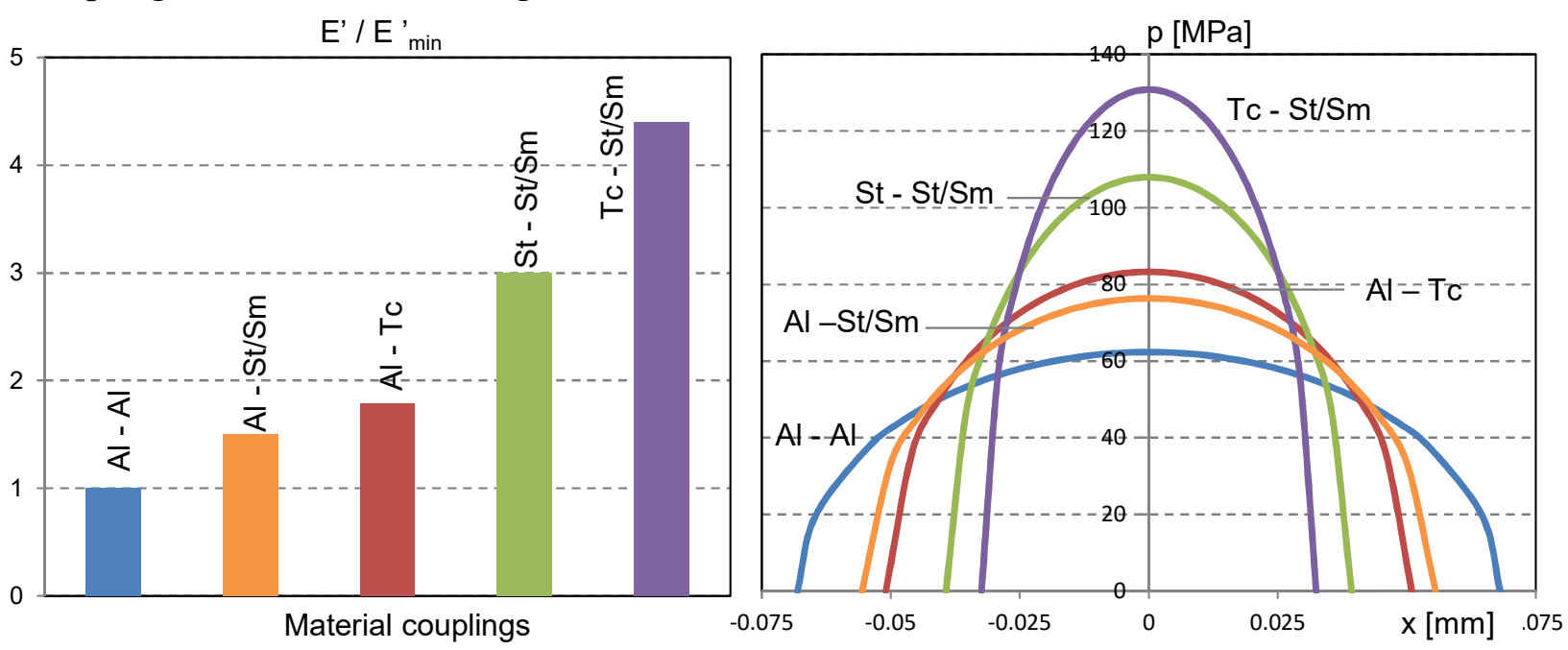

Figure 2: Left: Scaled equivalent modulus for the material couplings. Right: Hertzian pressure profiles.

Roughness. Another important feature of the tested couplings is their composite surface roughness, evaluated as

$$
R_{q}=\sqrt{R_{q c}^{2}+R_{q d}^{2}}
$$

where $R_{q c}$ and $R_{q d}$ are the root mean square roughness of cylinder and disc. The composite roughness of the tested samples is reported in Fig.3, separately for each material combination. It can be observed that $R_{q}$ values are within the intervals $0.36-2.05 \mu \mathrm{m}$ and $0.33-2.55 \mu \mathrm{m}$, for the Al-St and St-St couplings respectively. The minimum value $(0.13 \mu \mathrm{m})$ was reported for the St-Tc coupling. 

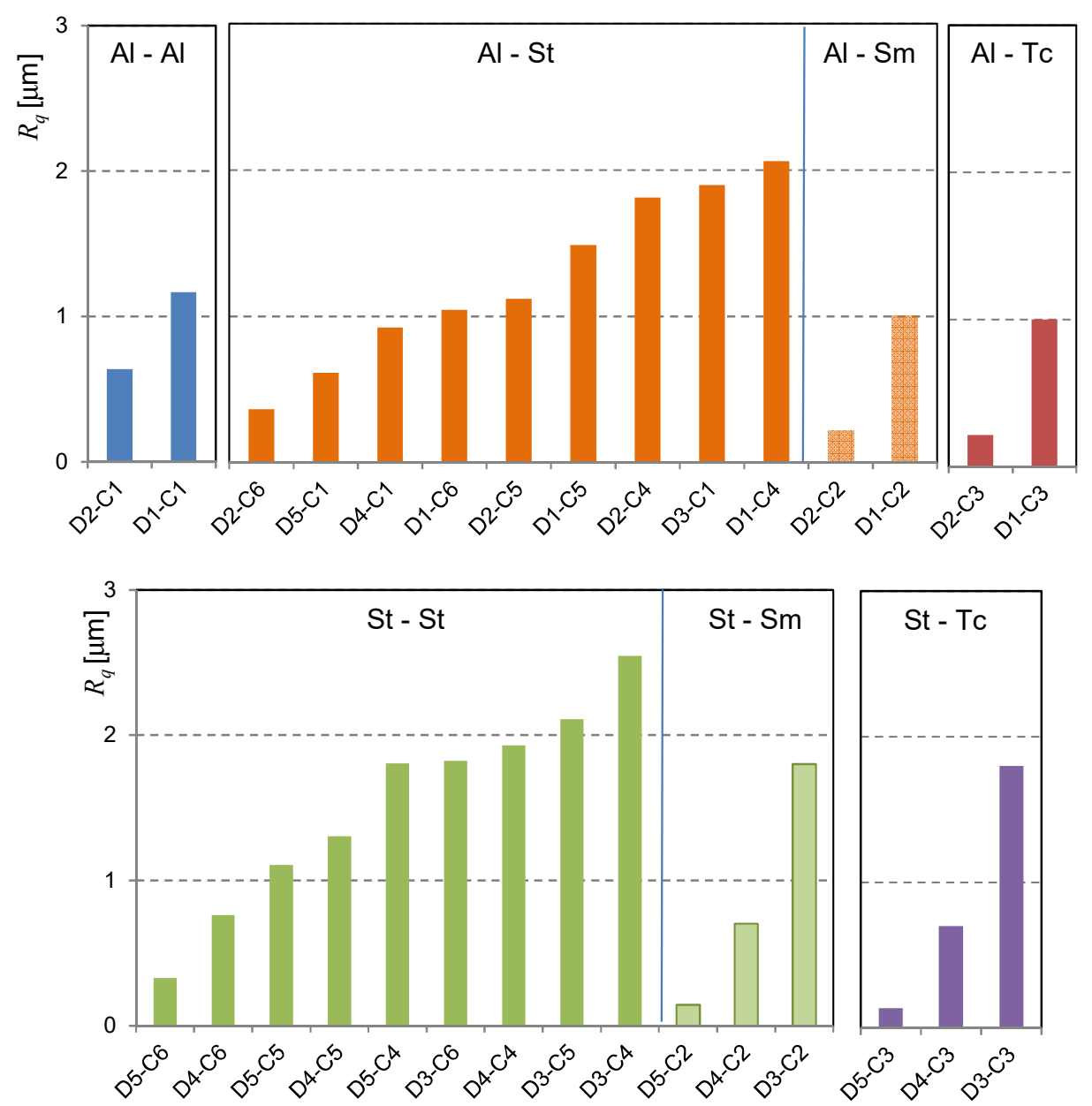

Figure 3: Composite roughness of the 36 couplings, distinguished for each material combination.

Lubrication. The main role in friction assessment is played by the lubrication characteristics. In the applied test conditions, the lubrication regime was piezo-viscous elastic in all cases so that the minimum $\left(h_{\min }\right)$ and central $\left(h_{\mathrm{c}}\right)$ film thickness can be calculated according to the following formulas $[7,8]$

$$
\begin{aligned}
& h_{\min }=2.66 \frac{\mu_{0}^{0.7} \alpha^{0.54} u^{0.7} R^{0.43}}{w^{0.13} E^{0.03}} \\
& h_{c}=2.922 \frac{\mu_{0}^{0.692} \alpha^{0.47} u^{0.692} R^{0.474}}{w^{0.166} E^{10.056}}
\end{aligned}
$$

It can be observed that such formulas were preferred to the most recent ones proposed in [3], because the latter ones provide unreasonable $h_{\min }>h_{\mathrm{c}}$ values, being fitted on higher values both of load and rolling speed.

The ratio between the minimum film thickness and the composite roughness provides the so called lambda ratio $\Lambda$

$$
\Lambda=\frac{h_{\min }}{R_{q}}
$$

used to identify the lubrication regime. As in the tested cases, for each material combination (i.e. for each $E^{\prime}$ ), the value of $\Lambda$ depends on the composite roughness $R_{q}$ and on the rolling speed $u$, in Fig.4 the maximum (dashed) and minimum (solid) $\Lambda$ curves for the material coupling are reported vs. $u$. 
Maximum values were obtained with the minimum composite roughness and vice versa. It can be observed that, since $\Lambda<1.2$ in all tests, mixed or mainly boundary lubrication regime occurred.

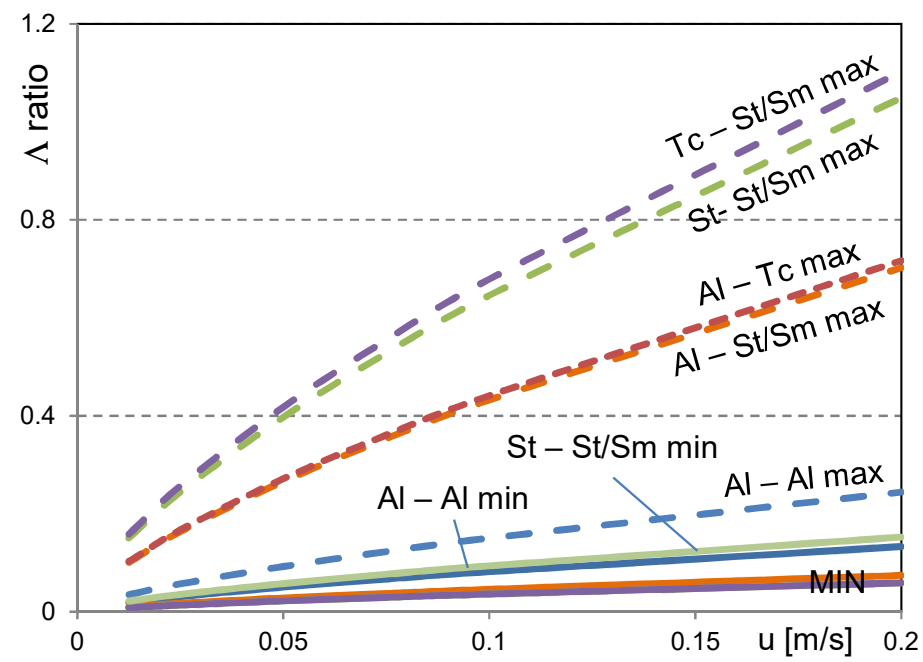

\begin{tabular}{|c|c|c|}
\hline \multirow{2}{*}{ Al-Al } & $\max$ & D2-C1 \\
\cline { 2 - 3 } & $\min$ & $\mathrm{D} 1-\mathrm{C} 1$ \\
\hline \multirow{2}{*}{ Al-St/Sm } & $\max$ & $\mathrm{D} 2-\mathrm{C} 2$ \\
\cline { 2 - 3 } & $\min$ & $\mathrm{D} 1-\mathrm{C} 4$ \\
\hline \multirow{2}{*}{ Tc-St/Sm } & $\max$ & $\mathrm{D} 5-\mathrm{C} 3$ \\
\cline { 2 - 3 } & $\min$ & $\mathrm{D} 3-\mathrm{C} 3$ \\
\hline \multirow{2}{*}{$\mathrm{Al}-\mathrm{Tc}$} & $\max$ & $\mathrm{D} 2-\mathrm{C} 3$ \\
\cline { 2 - 3 } & $\min$ & $\mathrm{D} 1-\mathrm{C} 3$ \\
\hline \multirow{2}{*}{ St-St/Sm } & $\max$ & $\mathrm{D} 5-\mathrm{C} 2$ \\
\cline { 2 - 3 } & $\min$ & $\mathrm{D} 3-\mathrm{C} 4$ \\
\hline
\end{tabular}

Figure 4: Lambda ratio minimum (solid) and maximum (dashed) curves distinguished for each material combination as a function of the rolling speed. Apart from Al-Al min curve, which is clearly identified, minimum curves for other materials (MIN) are almost overlapped. The table on the right indicates the coupling IDs used for the curves in the plot.

Friction. The friction coefficient $f$ is often evaluated from boundary to full film lubrication conditions by using a load sharing function $g(\Lambda)$ representing the portion of load supported by the full film contact. Several formulas for $g(\Lambda)$ can be found in literature, for instance the one reported in [9]

$$
g(\Lambda)= \begin{cases}0.84 \Lambda^{0.23} & \text { if } \Lambda \leq 2.1 \\ 1 & \text { if } \Lambda>2.1\end{cases}
$$

According to [10], once the load sharing factor is known, the friction coefficient $f$ can be calculated as

$$
f=f_{b}[1-g(\Lambda)]+f_{h}[g(\Lambda)]^{1.2}
$$

where $f_{h}$ and $f_{b}$ are the friction coefficients in the hydrodynamic (full fluid) and boundary lubrication conditions, respectively. The trends of the functions in Eqs.(6-7) are reported in Fig.5 for the range of $\Lambda$ of interest, according to Fig.4. Obviously, the reliability of the predicted friction coefficient strongly relies on the values attributed to $f_{h}$ and $f_{b}$. While the latter is usually considered constant (typically in the range $0.08-0.1$ ), the determination of $f_{h}$ includes several aspects. 

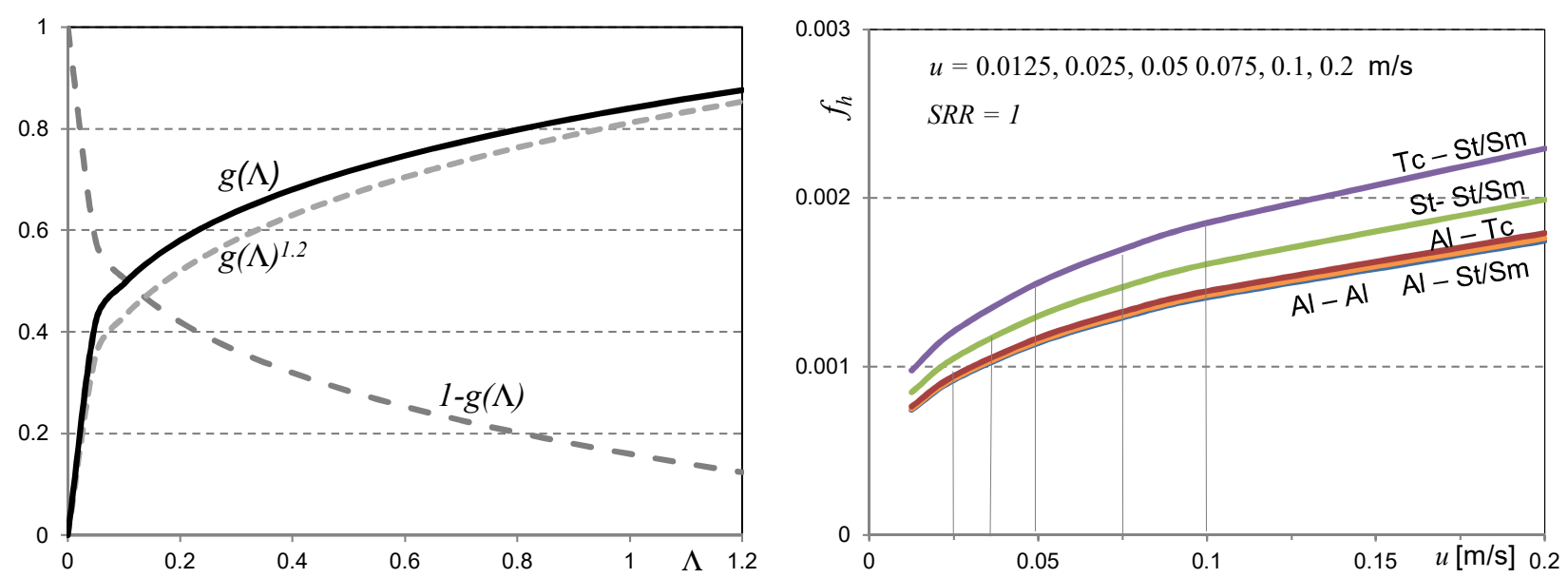

Figure 5: Left: plot of the functions in Eqs.(6-7) for evaluating the friction coefficient. Right: example of trends of $f_{h}$ for the tested material couplings $(S R R=1)$, according to Eq.(14). Al-Al, AlTc and Al-St/Tc are almost overlapped.

A mean value of $f_{h}$ can be evaluated by dividing the shear stress $\tau$ by the mean contact pressure $p_{m}$, i.e.

$$
f_{h}=\frac{\tau}{p_{m}}
$$

According to Hertz formulas, the mean contact pressure in linear contacts can be calculated as

$$
p_{m}=\sqrt{\frac{\pi w E^{\prime}}{32 R}}=\frac{\pi}{4} p_{\text {max }}
$$

while the shear stress $\tau$ depends on the adopted lubricant model. For example, in case of Newtonian fluid, it is simply

$$
\tau=\mu \dot{\gamma}
$$

where $\mu$ is the dynamic viscosity and $\dot{\gamma}$ the shear strain rate. In order to take into account the pressure effect on viscosity, as already hypothesized above, the Barus formula is assumed being

$$
\mu=\mu_{0} e^{\alpha p_{m}}
$$

As a first approximation, $\dot{\gamma}$ can be evaluated as the ratio of the difference between the velocities of the two points in contact, $\Delta u=u_{\mathrm{c}}-u_{\mathrm{d}}$, and the central film thickness $h_{c}$, that is

$$
\dot{\gamma}=\frac{\Delta u}{h_{c}}=\frac{\operatorname{SRR} u}{h_{c}}
$$

so that we can write:

$$
f_{h}=\frac{\mu_{0} e^{\alpha p_{m}} u}{p_{m} h_{c}} S R R
$$

Substituting Eq.(4) in Eq.(13), the following expression is obtained

$$
f_{h}=\left(\frac{1}{2.922} \frac{w^{0.166}}{R^{0.474}}\right)\left(\frac{\mu_{0}^{0.308}}{\alpha^{0.47}}\right)\left(\frac{e^{\alpha_{p_{m}}} E^{0.056}}{p_{m}}\right)\left(u^{0.308} S R R\right)
$$

where in the right term, four groups are highlighted by brackets: the first one depends on the geometry and on the load, the second one on the lubricant parameters, the third one on the material, lubricant and on the contact features, the latter on the kinematic conditions of the tests. Thus, for the present case, only the third term varies with the coupling remaining independent from the surface 
roughness. The trends of $f_{h}$ for the examined material combinations are reported in Fig.5 (right) as a function of the rolling speed $u$, for $S R R=1$. It can be observed that the difference between the curves increases with $u$ and all the curves for Aluminium couplings are almost overlapped. As $f_{h}$ is proportional to $S R R$, its trend for other test conditions $(S R R=0.25,0.5)$ can be obtained simply scaling plots in Fig.5. However, $f_{h}$ is very small (in the order of $10^{-3}$ ) with respect to the expected values of $f_{b}$ that the hydrodynamic contribution appears negligible for the cases in figure.

\section{Experimental Results.}

In order to provide an overview of the tested samples, in Fig. 6 three worn discs and one specimen are shown.

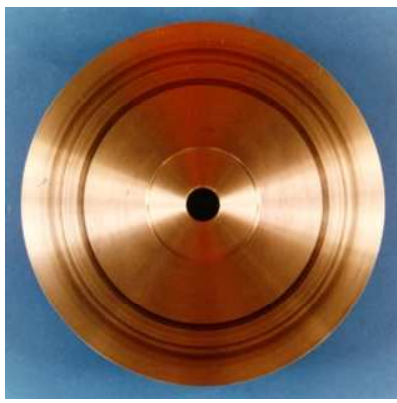

$\mathrm{D} 1-\mathrm{AL}_{1000}$

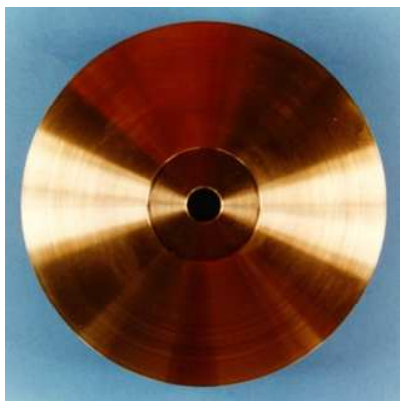

$\mathrm{D} 3-\mathrm{St}_{1800}$

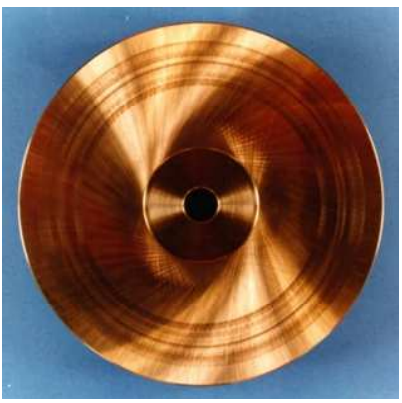

D4- $\mathrm{St}_{700}$

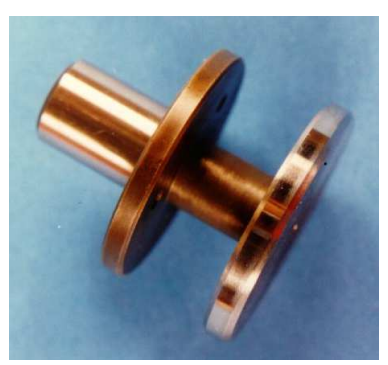

$\mathrm{C} 4-\mathrm{St}_{1800}$

Figure 6. Example of tested discs and specimen.

Friction Coefficient. An example of test results is reported in Figure 7, representing the friction coefficient vs. $u$, separately for the four material combinations, for the three SRR values. To limit the number of curves in each diagram, three couplings have been selected for each material combination, the ones with minimum, maximum and intermediate composite roughness $R_{\mathrm{q}}$.

Although the behaviour varies from material to material some general features can be observed: the friction coefficient tends to increase with $R_{\mathrm{q}}$ and $S R R$, while it decreases as $u$ increases. The decreasing behaviour of each curve clearly indicates the presence of boundary and mixed lubrication conditions. It can also be noted that, when the composite roughness is higher than $1 \mu \mathrm{m}$, the variation with $u$ is limited for St-St and St-Tc samples. In two cases, i.e. D5-C6 and D4-C3, the effect of $S R R$ is hardly visible. The resulting values of the friction coefficient mainly lie within the range $0.15-0.25$, apart from the St-Tc coupling with $R_{\mathrm{q}}=0.13 \mu \mathrm{m}$, presenting very low values (lower than 0.05) in particular for $u>0.05 \mathrm{~m} / \mathrm{s}$. For both the St-St and Al-St couplings, the samples with higher roughness are described by very similar curves and in some parts lower friction coefficient corresponds to higher roughness samples, which is not expected and difficult to explain. 

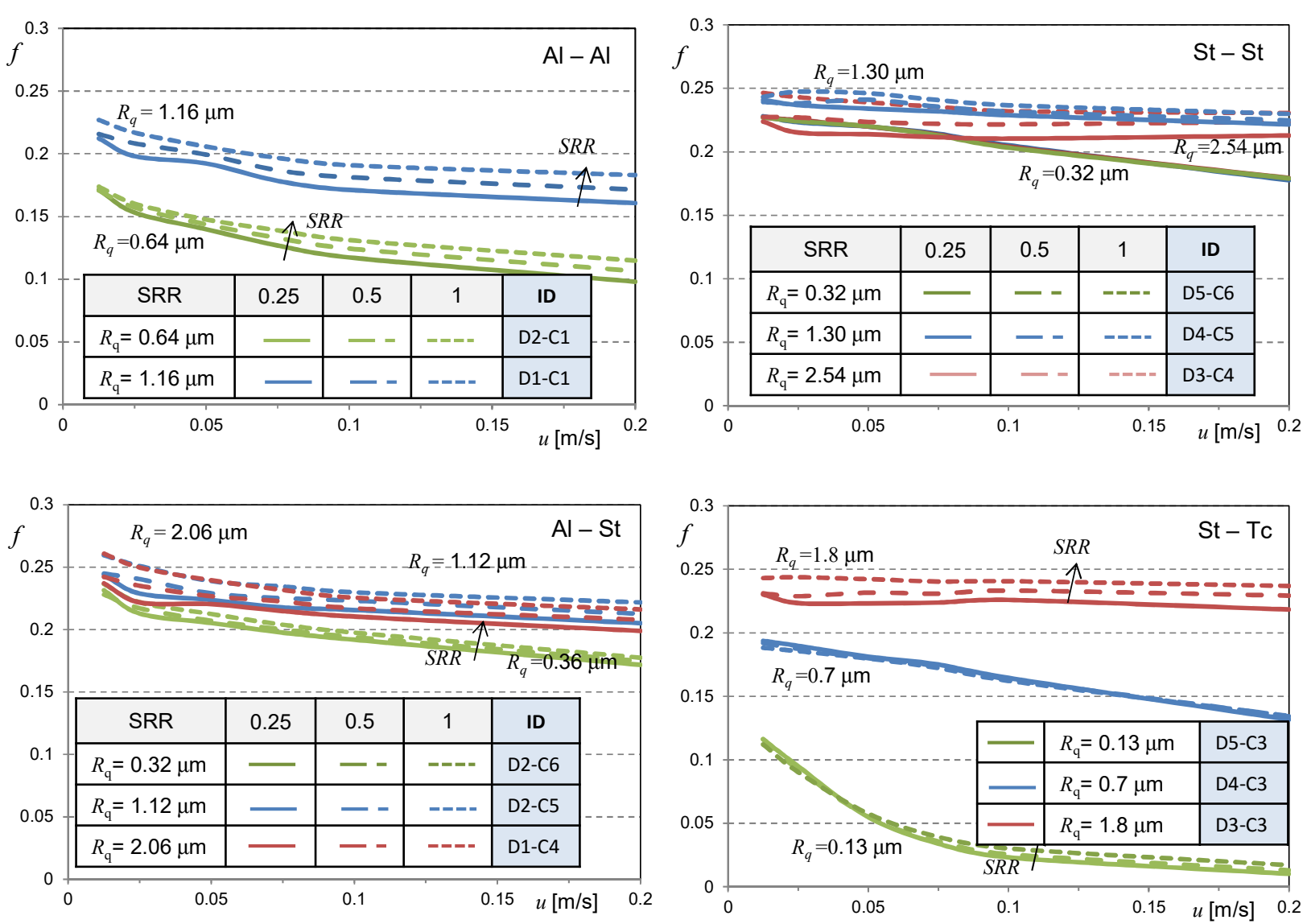

Figure 7. Experimental results of the friction coefficient for four material combinations. For each case, the couplings with minimum, maximum and intermediate composite roughness values have been selected for representation.

Another way of presenting results is reported in Fig.8 for the Al-St and St-St coupling, where the friction coefficient is plotted vs. $\Lambda$. In this way, the type of lubrication of the coupling is clearer, with respect to the representation of Fig.7. In particular, it can be noted that the two couplings with higher roughness were tested under very low lambda $\Lambda$ values $(\Lambda<0.15)$. Only for low roughness values, the lambda ratio increases to nearly 0.5 .
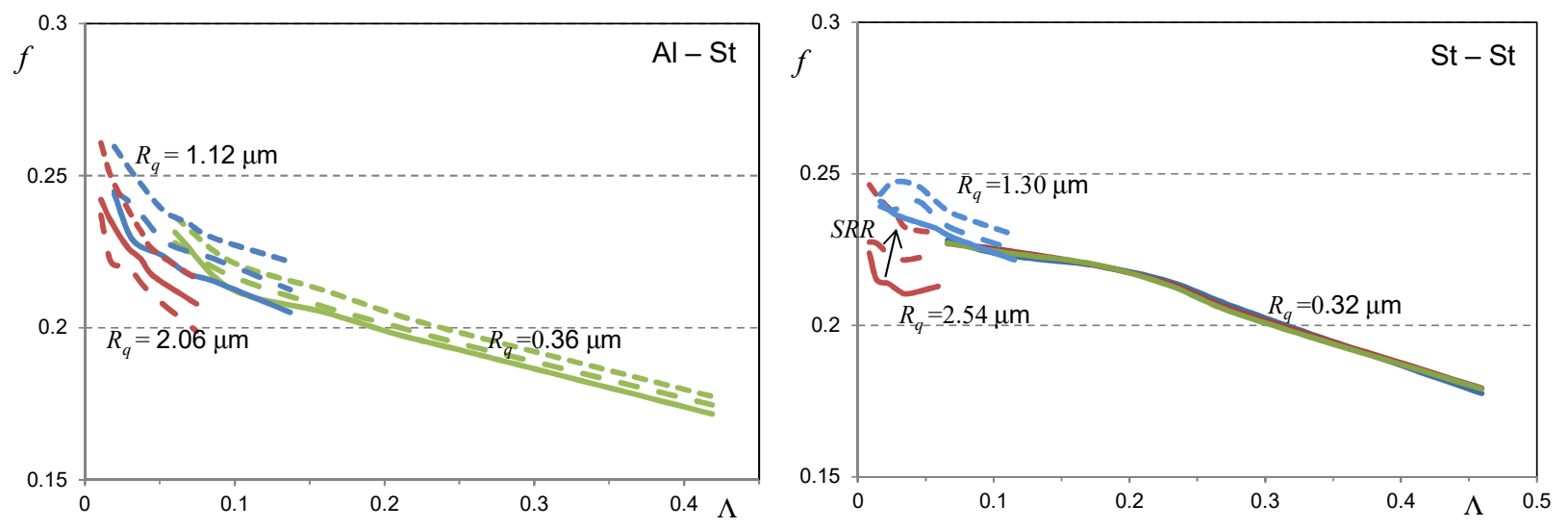

Figure 8. Representation of the friction coefficient vs. lambda ratio for two material couplings. Legenda in the correspondent plot in Fig.7.

Further interesting observations can be done comparing steel couplings with similar composite roughness, as shown in Fig.9 for $S R R=0.5$. While two couplings present a very similar behaviour, the third one shows much higher friction values, probably due to a different texture of the samples. 


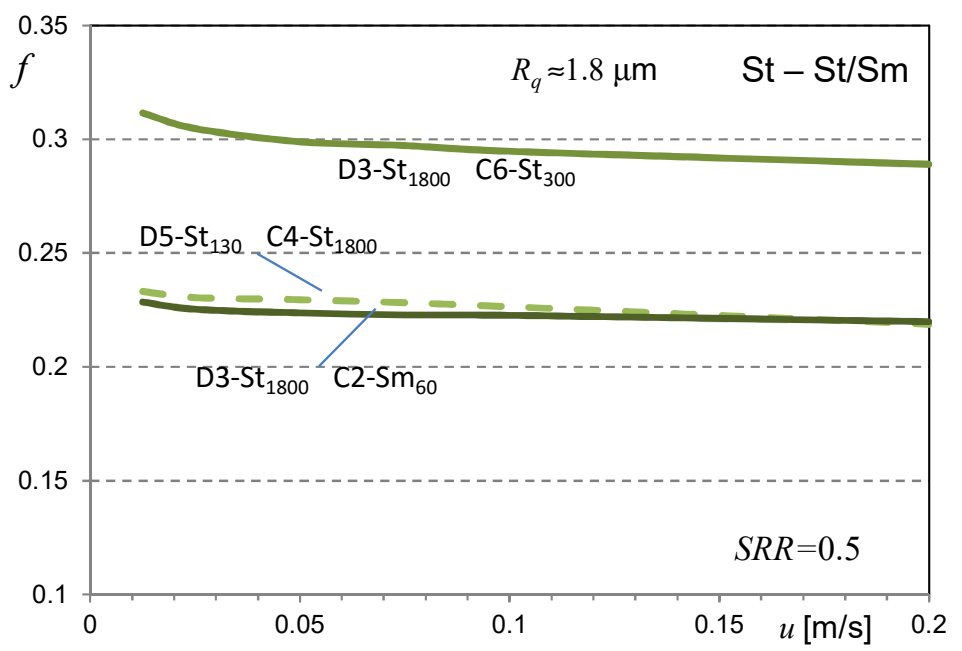

Figure 9. Representation of the friction coefficient vs. lambda ratio for steel couplings with similar composite roughness. Legenda in the correspondent plot in Fig.7. In this case the complete ID

(Table 1) of the samples is used to specify material and roughness of the elements.

Finally, it can be observed that the values of the friction coefficient, predicted according to Eq.(7) and the trends described in Fig.5, are about half the ones obtained from measurements. Moreover, in most cases the effect of $S R R$ in experimental results is higher than expected, even of orders of magnitudes, particularly for Al-Al, Al-St and St-Tc(D5-C3).

Worn Surface Analysis. In order to gain a wider comprehension of the phenomenon and of the previous results, the morphology of the surfaces and the wear effects were examined in depth by analysing the tracks left by the specimens on the discs. The shape of each track was observed by an optical microscope connected to a computer and by a stylus profilometer. Images acquired by the microscope were obtained by two magnification scales: one gives the complete track width ( $3 \mathrm{~mm})$ and the other one details of it. An example of the worn surface analysis is depicted in Figure 10 for the disc $\mathrm{D} 1-\mathrm{Al}_{1000}$; for each track, two pictures corresponding to both magnification scales are reported. The horizontal light lines, visible in the higher magnification images, correspond to the original tool marks of the disc; the contact of each specimen results in a more or less evident alteration of these marks on the relative track. The specimens $\mathrm{C} 2-\mathrm{Sm}_{60}$ and $\mathrm{C} 3-\mathrm{Tc}_{35}$ appear to have simply rounded the crests of the tooled profile, while $\mathrm{C} 4-\mathrm{St}_{1800}$ and even more $\mathrm{C} 6-\mathrm{St}_{300}$ and C5$\mathrm{St}_{1100}$ have markedly flattened them. The last two tracks present evident traces of adhesive wear, locally for specimen $\mathrm{C} 6-\mathrm{St}_{300}$ and extended for $\mathrm{C} 5-\mathrm{St}_{1100}$ where the initial tool marks are no more visible. The contact between disc $\mathrm{D} 1-\mathrm{Al}_{1000}$ and specimen $\mathrm{C} 2-\mathrm{Al}_{600}$, both of Aluminum, shows a typical phenomenon of material transfer with the consequent creation of a crater in the track. 


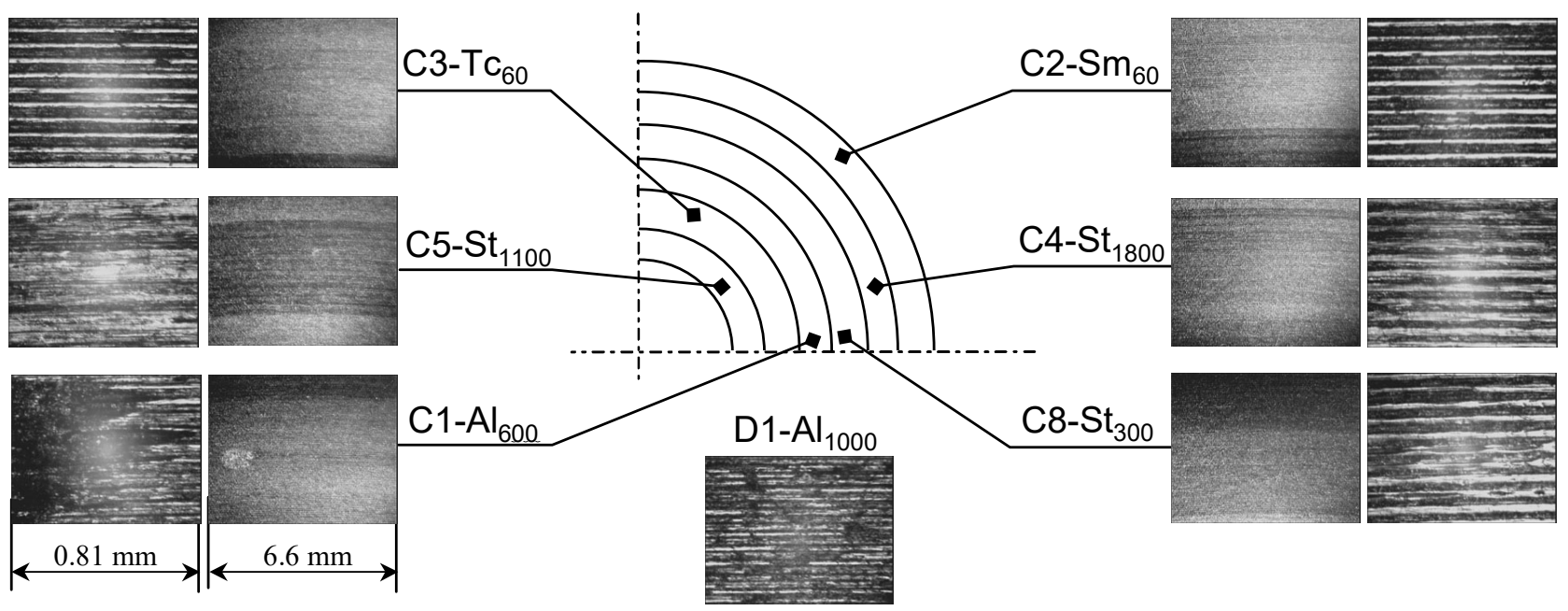

Figure 10. Analysis of disc $\mathrm{D} 1-\mathrm{Al}_{1000}$ by images.

In order to enrich image information, a quantitative estimate of the wear effects was carried out by evaluating $R_{q}$ values for the worn tracks, collected in Table 2 for each disc.

Table $2 R \mathrm{q}(\mu \mathrm{m})$ of worn tracks for each disc.

\begin{tabular}{|c|cccccc|}
\hline Coupling & $\mathrm{C} 1 \mathrm{Al}_{600}$ & $\mathrm{C} 2 \mathrm{Sm}_{60}$ & $\mathrm{C} 3 \mathrm{TcC}_{35}$ & $\mathrm{C} 4 \mathrm{St}_{1800}$ & $\mathrm{C} 5 \mathrm{St}_{100}$ & $\mathrm{C} \mathrm{St}_{300}$ \\
\hline $\mathrm{D} 1-\mathrm{Al}_{1000}$ & 0.95 & 0.99 & 0.98 & 0.40 & 0.30 & 0.85 \\
$\mathrm{D} 2-\mathrm{Al}_{250}$ & 0.25 & 0.2 & 0.2 & 0.21 & 0.28 & 0.26 \\
$\mathrm{D} 3-\mathrm{St}_{1800}$ & 1.80 & 1.80 & 1.80 & 1.80 & 1.80 & $1.80^{*}$ \\
$\mathrm{D} 4-\mathrm{St}_{700}$ & 0.62 & 0.66 & 0.57 & 0.65 & 0.59 & 0.53 \\
$\mathrm{D} 5-\mathrm{St}_{130}$ & 0.14 & 0.11 & 0.10 & 0.12 & 0.14 & $0.08^{*}$ \\
\hline
\end{tabular}

Similar analyses were completed also for the other discs; just to show results for a different material the case of the steel disc $\mathrm{D} 4-\mathrm{St}_{700}$ is reported in Figure 11. The contact tracks due to the tests are clearly visible and overlapped to the oblique lines of the initial texture, but with respect to the Aluminium disc, the tracks are less marked and wear debris can be observed.

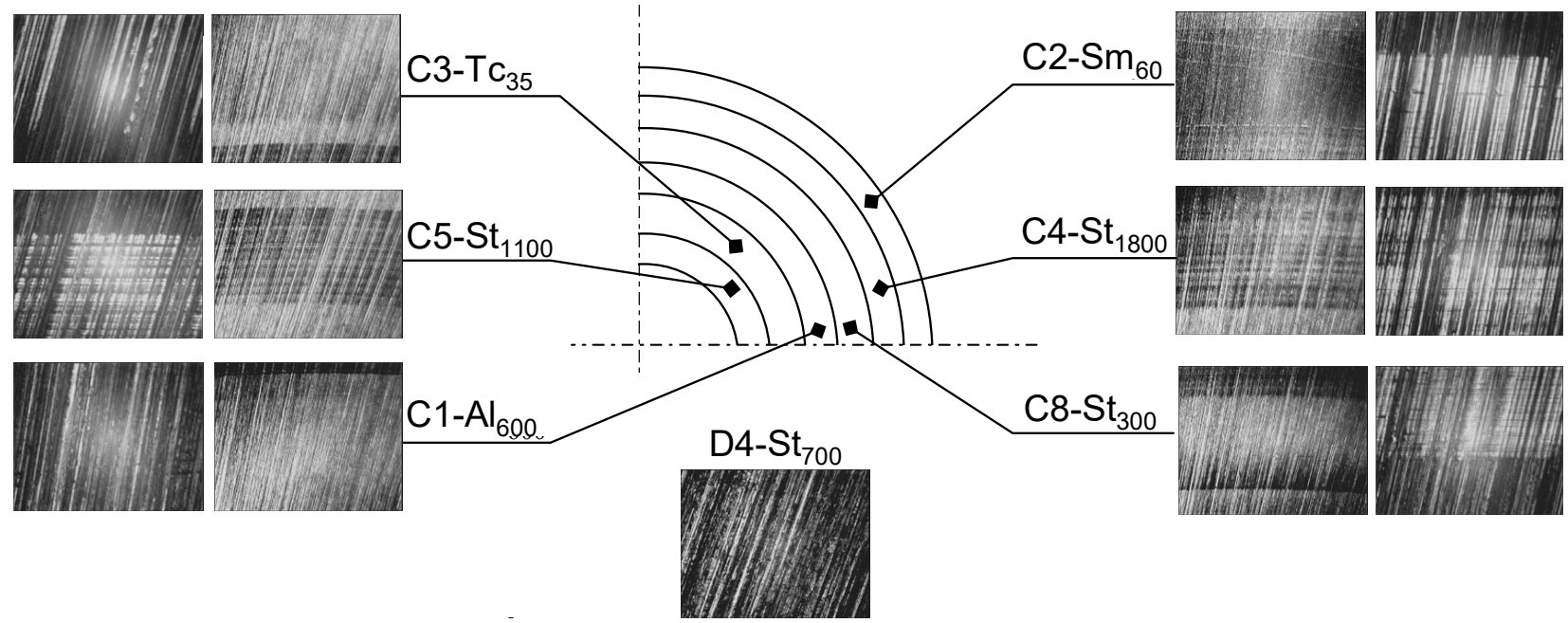

Figure 11. Analysis of disc D4-St 700 by images.

The pictures reported in Figures 10-11 can explain friction results shown in Figure 12, showing a complete summary of the tests result for the two discs. In the diagrams, the coefficient of friction is 
represented for each specimen and for each combination of $u$ and $S R R$. The horizontal axis reflects also the temporal sequence of the tests; for each specimen increasing speeds were imposed, combined with each $S R R$ value. As far Disc D1 is concerned (Fig. 12 left), it can be observed that stainless steel specimens' curves are all located in the upper area of the plot. In fact, the highest friction values correspond to the specimen $\mathrm{C} 5-\mathrm{St}_{1100}$, whose track is deeply plowed. The different behaviour of the other materials may be explained by the low roughness for the specimens $\mathrm{C} 2-\mathrm{Sm}_{60}$ and $\mathrm{C} 3-\mathrm{Tc}_{35}$, while for the aluminium one $\mathrm{C} 1-\mathrm{Al}_{600}$ an evident decrease of friction coefficient due to wear effects can be observed. A completely different behaviour stems from the contact of disc D4$\mathrm{St}_{700}$ with sample $\mathrm{C} 2-\mathrm{Al}_{600}$ which curve is at the top of Figure 12 right. The image in Figure 11 highlights the heavy wear of the specimen with the presence of a large number of wear debris. The curves of the other specimens are disposed decreasingly according to their roughness.
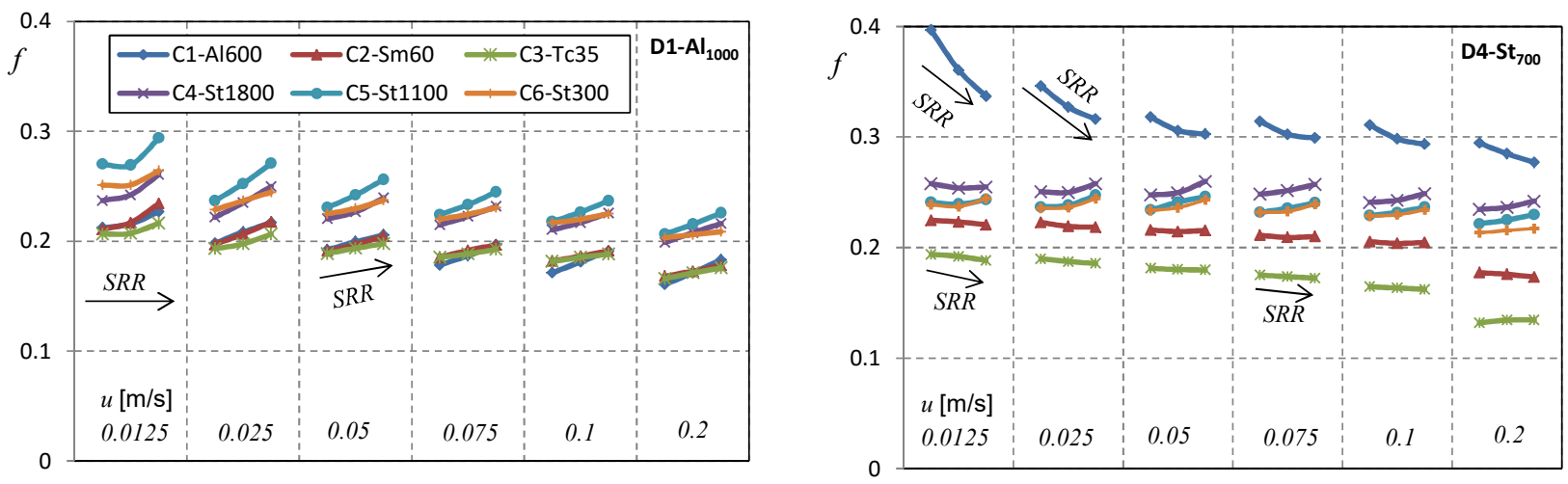

Figure 12. Friction coefficient for each test conducted with discs $\mathrm{D} 1-\mathrm{Al}_{1000}$ (left) and $\mathrm{D} 4-\mathrm{St}_{700}$ (right).

As already observed, $f$ is generally higher for higher $S R R$, while for the steel disc, $\mathrm{C} 1-\mathrm{Al}_{600}, \mathrm{C} 2$ $\mathrm{Sm}_{60}$ and $\mathrm{C} 3-\mathrm{Tc}_{35}$ specimens show an opposite trend, due to wear effects. These effects can be attributed mainly to the specimen for $\mathrm{C} 1-\mathrm{Al}_{600}$ while to the disc for $\mathrm{C} 2-\mathrm{Sm}_{60}$ and $\mathrm{C} 3-\mathrm{Tc}_{35}$ depending on the different hardness of the materials. This is confirmed also by the examination of the surfaces of the specimens before and after the tests. It may be worth observing that for each material the slope of $f$ remains almost constant for every speed step. It should be also noted that the maximum of the friction coefficient occurs when the specimen and the disc have similar values of roughness (specimen $\mathrm{C} 7-\mathrm{St}_{1100}$ with disc $\mathrm{A} 1-\mathrm{Al}_{1000}$ and $\mathrm{C} 2-\mathrm{Al}_{600}$ with $\mathrm{A} 4-\mathrm{St}_{700}$ ).

\section{Conclusions}

The contact between cylindrical specimens and the plane surface of several discs has been studied for different materials, surface roughness and texture. A theoretical background is presented to depict the contact features of each coupling and also to test friction predictive formulas.

The trends of the friction coefficient are analysed in different ways, versus the rolling speed and versus lambda, for enhancing the role of lubrication. As mainly boundary lubrication occurred, direct contact between asperities ensued and wear affected the tests, so the analysis is complicated by the continuous variations of the roughness and the morphology of the surfaces during each run. This was confirmed also by visual analysis of the worn surfaces.

Theoretical formulas predicted friction values about one half the ones measured experimentally; in particular they were not able to predict the role of different textures and also the influence of SRR on the friction coefficient is higher than expected. Such difference can be attributed to the very low values of the lambda ratio, suggesting an almost direct contact between asperities that causes also wear and variation of the surface morphology. Summing up, this study describes a procedure for analysing and maybe also designing properly a test campaign for friction evaluation, presents many results for different material and roughness combinations, and highlights future perspective for including features in predictive formulas for the assessment of the friction coefficient. 


\section{References}

[1] A.A. Pitenis, D. Dowson, W. Gregory Sawyer, Leonardo da Vinci's friction experiments: An old story acknowledged and repeated, Tribology Letters 56(3) (2014) 509-515. DOI: $10.1007 / \mathrm{s} 11249-014-0428-7$

[2] U. Besson, Historical Scientific Models and Theories as Resources for Learning and Teaching: The Case of Friction, Science and Education 22 (2013) 1001-1042. DOI 10.1007/s11191-0129502-4.

[3] M. Masjedi, M.M. Khonsari, Film thickness and asperity load formulas for line-contact elastohydrodynamic lubrication with provision for surface roughness, Journal of Tribology 134(1) (2012) art.011503. DOI: 10.1115/1.4005514.

[4] D. Zhu, Q.J. Wang, Effect of roughness orientation on the elastohydrodynamic lubrication film thickness, J Tribol Trans ASME 135 (2013), art. 031501. DOI: 10.1115/1.4023250

[5] E. Ciulli, Thermal effects of different kind influencing lubricated non-conformal contacts, Tribology International 59 (2013) 181-189. DOI: 10.1016/j.triboint.2012.05.007

[6] E. Ciulli, T. Draexl, K. Stadler, Film Thickness Analysis for EHL Contacts under Steady-State and Transient Conditions by Automatic Digital Image Processing, Advances in Tribology (2008) art. 325187. DOI: 10.1155/2008/325187

[7] B. J. Hamrock, D. Dowson, Isothermal Elastohdrodynamic Lubrication of Point Contacts, Part 2:, ellipticity parameter results, ASME Journal of Tribology 98 (1976) 223-229.

[8] B.J. Hamrock, Fundamentals of Fluid Film Lubrication, CRC press, New York, 2004.

[9] J. Castro, J. Seabra, Coefficient of friction in mixed film lubrication: gears versus twin-discs, Proc. IMechE Part J (221) (2007) 399-411. DOI: 10.1243/13506501JET257

[10] J. Castro, A. Campos, A. Sottomayor, J. Seabra, Friction Coefficient between Gear Teeth in Mixed Film Lubrication, in D. Dowson et al. (Eds), Life Cycle Tribology, Elsevier, Amsterdam, 2005, pp.525-533. DOI:10.1016/S0167-8922(05)80505-X 